\title{
VOLUME 85, 2004
}

$\begin{array}{llrllr}\text { No. } 1 & \text { January } & 1-136 & \text { No. } 7 & \text { July } & 929-1040 \\ \text { No. } 2 & \text { February } & 137-320 & \text { No. } 8 & \text { August } & 1041-1232 \\ \text { No. } 3 & \text { March } & 321-480 & \text { No. } 9 & \text { September } & 1233-1456 \\ \text { No. } 4 & \text { April } & 481-648 & \text { No. } 10 & \text { October } & 1457-1632 \\ \text { No. } 5 & \text { May } & 649-800 & \text { No. II } & \text { November } & 1633-1840 \\ \text { No. } 6 & \text { June } & 801-928 & \text { No. } 12 & \text { December } & 184 \mid-2032\end{array}$

NOTICE TO READERS. The search capability on the AMS Journals Online Web site (http://ams.allenpress.com) now allows for rapid, targeted identification of articles that match user-specified subject and author criteria. Because of this capability we are able to save considerable production time that would have otherwise been required to generate year-end subject indexes in print. Printed author indexes will continue to be provided in the final issue of the year for each journal. Readers are encouraged to browse AMS journals online to search titles and abstracts of articles referred to in the index below by visiting the AMS Web site (http:/l www.ametsoc.org).

Index to Contributions by Author

Abraham, James; Strapp, J. Walter; Fogarty, Christopher; Wolde, MENGISTU; Extratropical Transition of Hurricane Michael: An Aircraft Investigation, 1323-1339

Aвrams, ElLIot; Implementation and Refinement of Digital Forecasting Databases, 1667-1672

Ackerman, Thomas P.; see Diner, David J.; 1491-1501

Ackerman, Thomas P.; see Kahn, Ralph A.; 1511-1522

Ackerman, Thomas P.; Braverman, Amy J.; Diner, David J.; Anderson, Theodore L.; Kahn, Ralph A.; Martonchik, John V.; Penner, Joyce E.; Rasch, Philip J.; Wielicki, Bruce A.; Yu, Bin; Integrating and Interpreting Aerosol Observations and Models within the PARAGON Framework, 1523-1533

Albright, Mark; see VAUGHAN, JosePH; 549-561

Alessandri, A.; see Palmer, T. N.; 853-872

Alexander, Lisa; see Page, Cher M.; 1483-1489

ANdersen, U.; see PALmer, T. N.; 853-872

Anderson, Kenneth; Brooks, Barbara; Caffrey, Peter; Clarke, Antony; Cohen, Leo; Crahan, Katie; Davidson, Kenneth; De Jong, Arie; De Leeuw, Gerrit; Dion, Denis; Doss-Hammel, Stephen; Frederickson, Paul; Friehe, Carl; Hristov, Timohir; Khelif, Djamal; Moerman, Marcel; Reid, Jeffery S.; Reising, Steven; Smith, Michael; Terrill, Eric; Tsintikidis, Dimitris; The RED Experiment: An Assessment of Boundary Layer Effects in a Trade Winds Regime on Microwave and Infrared Propagation over the Sea, 1355-1365

Anderson, Martha C.; see Diak, George R.; 65-78

Anderson, Theodore L.; see Diner, David J.; 1491-1501

Anderson, Theodore L.; see SeInfeld, John H.; 1503-1509

Anderson, Theodore L.; see ACKerman, Thomas P.; 1523-1533

Anderson, Theodore L.; see Diner, David J.; 1535-1548

AndRetta, Marco; see Rotach, Mathias W.; 1367-1385

ANTHES, Richard A.; see Windham, Thomas L.; 42-47

Antonelli, P.; Review of Remote Sensing of Atmosphere and Ocean from Space: Models, Instruments and Techniques, 1595

ARImoto, Richard; see SeINFELD, John H.; 367-380

Arsenault, K.; see Rodell, M.; 381-394

Arunachalam, Sarav; see McHenry, John N.; 525-548

Assel, Raymond A.; Quinn, Frank H.; Sellinger, Cynthia E.; Hydroclimatic Factors of the Recent Record Drop in Laurentian Great Lakes Water Levels, 1143-1151

Atkins, Nolan; see Davis, Christopher; 1075-1093

ATLAS, D.; BANDEEN-RoCHE, K.; "William Reid Bandeen, 19262004," obituary, 1604

Aune, R. M.; LewIS, J. M.; WadE, G. S.; "William H. Raymond, 1941-2003," obituary, 770

Averill, Clare; see Garay, Michael J.; 1585-1594

BaArs, Jeffrey A.; see Fischer, KenNeTh W.; 725-732

BACHMEIER, SCOTt; see Mostek, ANTHony; 823-829
Baggett, Kevin C.; see Huang, Hung-Lung; 159-161

Bales, R. C.; Liverman, D. M.; Morehouse, B. J.; Integrated Assessment as a Step Toward Reducing Climate Vulnerability in the Southwestern United States, 1727-1734

BANDEEN-RoChe, K.; see Atlas, D.; obituary, 1604

BARKeR, Llyle J., III; see LANG, Timothy J.; 1107-1125

Bartels, Diana; see Davis, Christopher; 1075-1093

Bates, Timothy S.; see Seinfeld, John H.; 367-380

BAUMGARDNER, D.; see WENDISCH, M.; 89-91

BAUMGARDNER, D.; see WENDISCH, M.; ES1-ES8 (BAMS Online, January)

Baumgardner, DarReL; see DabBerdT, W ALter F.; 563-586

Baumgardner, Darrel; see Bretherton, Christopher S.; 967-977

Bedient, T.; see Howcroft, J. G.; obituary, 110

BeItLer, Brian A.; see W ARner, Thomas T.; 709-716

Benedict, S.; see LAWFord, R. G.; 1917-1930

Benedict, Sam; see Bollasina, Massimo; 1001-1004

Beniamin, Stanley G.; Schwartz, Barry E.; Szoke, Edward J.; Косн, Steven E.; The Value of Wind Profiler Data in U.S. Weather Forecasting, 1871-1886

Bentamin, Stanley G.; Schwartz, Barry E.; Szoke, Edward J.; КосH, STeven E.; The Value of Wind Profiler Data in U.S. Weather Forecasting: Case Studies, ES21-ES29 (BAMS Online, December)

Bennetr, S.; "Michael Anderson Roberts, Jr., 1947-2004," obituary, 1607

Bentamy, A.; see Curry, J. A.; 409-424

BETTS, ALAN K.; Understanding Hydrometeorology Using Global Models, 1673-1688

Beven, John L., II; see Landsea, Christopher W.; 1699-1712

Bikos, Dan; see Mostek, ANTHony; 823-829

BinKOшsкI, F.; Review of Handbook of Atmospheric Science: Principles and Applications, 1391

BlanCHARD, D. C.; Review of The Snowflake: Winter's Secret Beauty, 1387

BloeminK, H.; see Crewell, S.; 1565-1584

BOGDAN, T. J.; Review of The Maunder Minimum and the Variable Sun-Earth Connection, 1767

Bollasina, Massimo; Benedict, Sam; The Role of the Himalayas and the Tibetan Plateau within the Asian Monsoon System, 1001-1004

Bosart, Lance; see Davis, Christopher; 1075-1093

Bosart, Lance F.; see Davis, Christopher, A.; 1657-1662

BÖSENBERG, Jens; see Diner, DaVID J.; 1491-1501

BÖSENBERG, JENS; see KaHN, RALPH A.; 1511-1522

Bösenberg, Jens; see Diner, David J.; 1535-1548

Bosilovich, M.; see Rodell, M.; 381-394

BOURASSA, M. A.; see CURRY, J. A.; 409-424 
Bourassa, Mark A.; see Smith, Shawn R.; 979-994

BOURRAS, D.; see CurRY, J. A.; 409-424

Bowers, James F.; see Warner, Thomas T.; 709-716

Bowman, Clint; see Vaughan, Joseph; 549-561

Bowman, David; see Powell, Mark; 845-851

BOyer, Mike; see Vaughan, Joseph; 549-561

Boyle, James S.; see Phillips, Thomas J.; 1903-1915

Bradley, E. F.; see CurRY, J. A.; 409-424

Braverman, Amy J.; see Diner, David J.; 1491-1501

Braverman, Amy J.; see Ackerman, Thomas P.; 1523-1533

Brechtel, Frederick J.; see Seinfeld, John H.; 367-380

Brenguier, J.-L.; see WendisCh, M.; 89-91

Brenguier, J.-L.; see Wendisch, M.; ES1-ES8 (BAMS Online, January)

Brennan, Michael J.; Lackmann, Gary M.; Koch, Steven E.; The Impact of a Split-Front Rainband on Appalachian Cold-Air

Damming Erosion, 935-939

Bretherton, Christopher S.; UtTal, Taneil; Fairall, Christopher W.; Yuter, Sandra E.; Weller, Robert A.; Baumgardner, Darrel; Comstock, Kimberly; WoOd, Robert; Raga, Graciela B.; The EPIC 2001 Stratocumulus Study, 967-977

Bretherton, Christopher S.; see Raymond, David J.; 1341-1354

Brey, James A.; see Geer, Ira W.; 37-41

Bringi, V. N.; see Lang, Timothy J.; 1107-1125

Broad, Kenneth; see Orlove, Benjamin S.; 1735-1743

Broad, Kenneth; see Orlove, Benjamin S.; ES19-ES20 (BAMS

Online, November)

Brooks, Barbara; see Anderson, Kenneth; 1355-1365

BRooks, Harold E.; Tornado-Warning Performance in the Past and Future: A Perspective from Signal Detection Theory, 837-843

BRUNKE, M.; see CURRY, J. A.; 409-424

Brunner, R. D.; see LynCH, A. H.; 209-221

Bryan, GeOrge; see Davis, Christopher; 1075-1093

Caffrey, Peter; see Anderson, Kenneth; 1355-1365

Cahill, Thomas A.; see Seinfeld, John H.; 367-380

CaI, Huaqing; see Wakimoto, Roger M.; 1095-1106

Calanca, Pierluigi; see Rotach, Mathias W.; 1367-1385

Cantelaube, P.; see Palmer, T. N.; 853-872

Caracena, F.; “Adrian Marroquin, 1934-2003," obituary, 1016

Carbone, R. E.; see Fritsch, J. Michael; 955-965

Carmichael, Gregory; see Dabberdt, W alter F.; 563-586

Carmichael, Gregory R.; see Seinfeld, John H.; 367-380

Carrasco, Nick; see Powell, Mark; 845-851

Carroll, Mary AnNe; see Dabberdt, Walter F.; 563-586

Castro, S.; see Curry, J. A.; 409-424

Cattani, Elsa; see Rosenfeld, Daniel; 873-881

Cederwall, Richard T.; see Phillips, Thomas J.; 1903-1915

Chambers, Lynda E.; see Page, Cher M.; 1483-1489

Chandrasekar, V.; see Lang, Timothy J.; 1107-1125

Changnon, David; Improving Outreach in Atmospheric Sciences: Assessment of Users of Climate Products, 601-606

Changnon, David; Improving Outreach in Atmospheric Sciences: Assessment of Users of Climate Products, ES11-ES13 (BAMS Online, April)

Charlson, Robert J.; see Diner, David J.; 1491-1501

Charlson, Robert J.; see Seinfeld, John H.; 1503-1509

Charlson, Robert J.; see Kahn, Ralph A.; 1511-1522

Charlson, Robert J.; see Diner, David J.; 1535-1548

Chenoweth, Michael; Landsea, Christopher; The San Diego Hurricane of 2 October 1858, 1689-1697

Chol, Youngeun; see PAge, Cher M.; 1483-1489

Chou, S. H.; see Curry, J. A.; 409-424

Christen, Andreas; see Rotach, Mathias W.; 1367-1385

Cieslik, Stanislaw; see Rotach, Mathias W.; 1367-1385

Cifelli, Robert; see Raymond, David J.; 1341-1354

Clarke, Antony; see Anderson, Kenneth; 1355-1365

Clarke, Antony D.; see Seinfeld, John H.; 367-380

Clayson, C. A.; see Curry, J. A.; 409-424
Coats, Carlie J., Jr.; see McHenry, John N.; 525-548

COE, H.; see WENDISCH, M.; 89-91

CoE, H.; see Wendisch, M.; ES1-ES8

Cohen, Leo; see Anderson, Kenneth; 1355-1365

Cohen, Ronald; see Dabberdt, Walter F.; 563-586

Collingwood, Alice; see Vaughan, Joseph; 549-561

Collins, Dean A.; see Page, Cher M.; 1483-1489

Collins, William D.; see Diner, David J.; 1491-1501

Collins, William D.; see Diner, David J.; 1535-1548

COMSTOCK, Kimberly; see Bretherton, Christopher S.; 967-977

Conant, William C.; see Seinfeld, John H.; 367-380

Coniglio, Michael; see Davis, Christopher; 1075-1093

ConNolly, Richard; see Rotach, Mathias W.; 1367-1385

Cope, A.; Review of The Philadelphia Area Weather Book: Including Delaware, the Poconos, and the New Jersey Shore, 1769

Cormier, R.; Grantham, D.; Sissenwine, M.; "Norm Sissenwine, 1917-2004," obituary, 1166

Cosgrove, B.; see Rodel., M.; 381-394

Cotton, William; see Davis, Christopher; 1075-1093

Crahan, Katie; see Anderson, Kenneth; 1355-1365

Crewell, S.; Bloemink, H.; Feijt, A.; Garcia, S. G.; Jolivet, D.; Krasnov, O. A.; van Lammeren, A.; Löhnert, U.; van Meijgaard, E.; Meywerk, J.; Quante, M.; Pfeilsticker, K.; SChmidt, S.; Scholl, T.; Simmer, C.; Schröder, M.; Trautmann, T.; Venema, V.; Wendisch, M.; WilléN, U.; The BALTEX BRIDGE

Campaign: An Integrated Approach for a Better Understanding of Clouds, 1565-1584

Croft, P. J.; see Pandya, R. E.; 425-430

Cullen, H.; Review of Floods, Droughts, and Climate Change, 437

Curry, J. A.; see LynCH, A. H.; 209-221

Curry, J. A.; Bentamy, A.; Bourassa, M. A.; Bourras, D.; Bradley, E. F.; Brunke, M.; Castro, S.; Chou, S. H.; Clayson, C. A.; Emery, W. J.; Eymard, L.; Fairall, C. W.; Kubota, M.; Lin, B.; Perrie, W.; Reeder, R. A.; Renfrew, I. A.; Rossow, W. B.; SchulZ, J.; SMIth, S. R.; Webster, P. J.; WICK, G. A.; ZenG, X.; SEAFLUX, 409-424

Dabberdt, Walter F.; Carroll, Mary Anne; Baumgardner, Darrel; Carmichael, Gregory; Cohen, Ronald; Dye, Tim; Ellis, James; Grell, Georg; Grimmond, Sue; Hanna, Steven; Irwin, John; Lamb, Brian; Madronich, Sasha; McQueen, JefF; Meagher, James; Odman, Talat; Pleim, Jonathan; Schmid, Hans Peter; WestPhal, Douglas L.; Meteorological Research Needs for Improved Air Quality Forecasting: Report of the 11th Prospectus Development Team of the U.S. Weather Research Program, 563-586

Daniel, John S.; see Solomon, Susan; 1273-1288

DaveY, M.; see PALMER, T. N.; 853-872

Davidson, Kenneth; see Anderson, Kenneth; 1355-1365

Davies, James E.; see Huang, Hung-Lung; 159-161

DAVIES, Roger; see Diner, DAVID J.; 1491-1501

Davies, Roger; see Seinfeld, John H.; 1503-1509

Davies, Roger; see Garay, Michael J.; 1585-1594

Davis, Christopher; Atkins, Nolan; Bartels, Diana; Bosart, Lance; Coniglio, Michael; Bryan, George; Cotton, William; Dowell, David; JeWETt, Brian; Johns, RoberT; Jorgensen, David; KNIEvel, Jason; Knupp, Kevin; Lee, Wen-Chau; McFarquhar, Gregory; Moore, James; Przybylinski, Ron; Rauber, Robert; Smull, Bradley; Trapp, Robert; Trier, Stanley; Wakimoto, Roger; Weisman, Morris; Ziegler, Conrad; The Bow Echo and MCV Experiment: Observations and Opportunities, 1075-1093

Davis, Christopher A.; Bosart, Lance F.; The TT Problem:

Forecasting the Tropical Transition of Cyclones, 1657-1662

de Boer, Agatha M.; see Nof, Doron; 79-87

DeGaetano, Arthur T.; see Hubbard, Kenneth G.; 811-812

De Jong, Arie; see Anderson, Kenneth; 1355-1365

DÉlécluse, P.; see Palmer, T. N.; 853-872

De Leeuw, Gerrit; see Anderson, Kenneth; 1355-1365

Della-Marta, Paul; see Page, Cher M.; 1483-1489 
Demoz, Belay B.; see Weckwerth, Tammy M.; 253-277

DÉQuE, M.; see Palmer, T. N.; 853-872

Derezotes, Paul N.; Making the Jump to the Private Sector, 173176

Detwiler, ANdRew; see LANG, Timothy J.; 1107-1125

De Wekker, Stephan F. J.; see Rotach, Mathias W.; 1367-1385

DeWolf, Rebecca L.; see Diak, George R.; 65-78

Diak, George R.; MeciKalski, John R.; Anderson, Martha C.; Norman, John M.; Kustas, William P.; Torn, Ryan D.; DeWolf, REBECCA L.; Estimating Land Surface Energy Budgets from Space: Review and Current Efforts at the University of Wisconsin-Madison and USDA-ARS, 65-78

Diez, E.; see Palmer, T. N.; 853-872

Di Girolamo, P.; see Gérard, E.; 237-251

Diner, David J.; Ackerman, Thomas P.; Anderson, Theodore L.; Bösenberg, Jens; Braverman, Amy J.; Charlson, Robert J.; Collins, William D.; Davies, Roger; Holben, BRent N.; Hostetler, Chris A.; Kahn, Ralph A.; MartonchiK, John V.; Menzies, Robert T.; Miller, Mark A.; Ogren, John A.; Penner, Joyce E.; Rasch, Philip J.; Schwartz, Stephen E.; Seinfeld, John H.; Stephens, Graeme L.; Torres, Omar; Travis, Larry D.; Wielicki, Bruce A.; Yu, Bin; PARAGON: An Integrated Approach for Characterizing Aerosol Climate Impacts and Environmental Interactions, 1491-1501

Diner, David J.; see Seinfeld, John H.; 1503-1509

Diner, DaVID J.; see KaHN, RalPH A.; 1511-1522

Diner, David J.; see Ackerman, Thomas P.; 1523-1533

Diner, David J.; Menzies, Robert T.; Kahn, Ralph A.; Anderson, Theodore L.; Bösenberg, Jens; Charlson, Robert J.; Holben, Brent N.; Hostetler, Chris A.; Miller, Mark A.; Ogren, John A.; Stephens, Graeme L.; Torres, Omar; Wielicki, Bruce A.; Rasch, Philip J.; Travis, Larry D.; Collins, William D.; Using the PARAGON Framework to Establish an Accurate, Consistent, and Cohesive Long-Term Aerosol Record, 1535-1548

Dion, Denis; see Anderson, KenNeth; 1355-1365

Doblas-Reyes, F. J.; see Palmer, T. N.; 853-872

Dodge, James C.; see Huang, Hung-Lung; 159-161

Dodge, Peter P.; see Landsea, Christopher W.; 1699-1712

Doesken, Nolan; see Lang, Timothy J.; 1107-1125

Doherty, Sarah J.; see Seinfeld, John H.; 367-380

Doss-Hammel, StePhen; see Anderson, Kenneth; 1355-1365

Dowell, David; see Davis, Christopher; 1075-1093

Doyle, James D.; Durran, Dale R.; Recent Developments in the

Theory of Atmospheric Rotors, 337-342

DreILING, V.; see WENDISCH, M.; 89-91

Dreiling, V.; see WENDISCH, M.; ES1-ES8 (BAMS Online, January)

DueÑas, E. N.; see Rossow, W. B.; 167-172

Dunion, Jason P.; VeLden, Christopher S.; The Impact of the Saharan Air Layer on Atlantic Tropical Cyclone Activity, 353365

Dunion, Jason P.; see LandSEA, Christopher W.; 1699-1712

DupignY-Giroux, L.-A.; Review of New England Weather, New England Climate, 754

Durran, Dale R.; see Doyle, James D.; 337-342

Dye, Tim; see DabBerdT, Walter F.; 563-586

Ebi, Kristie L.; Teisberg, Thomas J.; Kalkstein, Laurence S.; Robinson, LaWrence; WeIHER, Rodney F.; Heat Watch/Warning Systems Save Lives: Estimated Costs and Benefits for Philadelphia 1995-98, 1067-1073

EHRET, G.; see GÉrARD, E.; 237-251

Ellis, James; see DabBerdt, Walter F.; 563-586

EMERY, W. J.; see CurRY, J. A.; 409-424

ENTIN, J. K.; see Rodell, M.; 381-394

Epstein, Paul R.; McCarthy, James J.; Assessing Climate Stability, 1863-1869

Esbensen, STEVen K.; see Raymond, David J.; 1341-1354

EsHel, G.; Review of Climate Development and History of the North Atlantic Realm, 750
ETTer, P. C.; Review of Underwater Acoustic Modeling and Simulation (Third Edition), 435

Eymard, L.; see CurRY, J. A.; 409-424

Fairall, C. W.; see CurRY, J. A.; 409-424

Fairall, Christopher W.; see Bretherton, Christopher S.; 967-977

Feddersen, H.; see Palmer, T. N.; 853-872

FeiJT, A.; see Crewell, S.; 1565-1584

Feltz, Wayne F.; see WeCKWERTh, Tammy M.; 253-277

FENG, Song; see Hu, QI; 29-31

FerreE, John; see Mostek, Anthony; 823-829

FIEBIG, M.; see WeNDISCH, M.; 89-91

FIEBIG, M.; see WENDISCH, M.; ES1-ES8 (BAMS Online, January)

Figueroa-Kaminsky, Cristiana; see Vaughan, Joseph; 549-561

Fiorino, Michael; see Phillips, Thomas J.; 1903-1915

Fischer, Kenneth W.; Witiw, Michael R.; BaArs, Jeffrey A.; OKe,

T. R.; Atmospheric Laser Communication: New Challenges for Applied Meteorology, 725-732

Flamant, Cyrille; see Weckwerth, Tammy M.; 253-277

Flatau, Piotr J.; see Seinfeld, John H.; 367-380

Fogarty, Christopher; see Abraham, James; 1323-1339

Formenti, P.; see Wendisch, M.; 89-91

FormentI, P.; see Wendisch, M.; ES1-ES8 (BAMS Online, January)

Frankin, James L.; see LandSea, Christopher W.; 1699-1712

Frederickson, PaUl; see ANDERSON, KenNeth; 1355-1365

Frei, Chris; see Vaughan, Joseph; 549-561

Friedman, H. A.; see PANDYA, R. E.; 425-430

Friehe, Carl; see Anderson, Kenneth; 1355-1365

Fritsch, J. Michael; Carbone, R. E.; Improving Quantitative

Precipitation Forecasts in the Warm Season: A USWRP

Research and Development Strategy, 955-965

Galmarini, Stefano; see Rotach, Mathias W.; 1367-1385

GARAND, L.; see GÉrard, E.; 237-251

Garay, Michael J.; Davies, Roger; Averill, Clare; Westphal, James A.; Actinoform Clouds: Overlooked Examples of Cloud Self-Organization at the Mesoscale, 1585-1594

GarCla, S. G.; see Crewell, S.; 1565-1584

Geer, Ira W.; Mills, Elizabeth W.; Moran, Joseph M.; Weinbeck, Robert S.; Porter, William A.; Harris, JASPer L.; Brey, James A.; Enhancing Diversity in the Geosciences through National Dissemination of the AMS Online Weather Studies Distance Learning Course, 37-41

Geerts, Bart; see WeCKWerth, Tammy M.; 253-277

Gentilcore, Anastasia N.; see Tselioudis, George; 830-835

Gérard, E.; Tan, D. G. H.; Garand, L.; Wulfmeyer, V.; Ehret, G.; DI Girolamo, P.; Major Advances Foreseen in Humidity Profiling from the Water Vapour Lidar Experiment in Space (WALES), 237-251

Gilhousen, David; see Powell, Mark; 845-851

Gilroy, Mike; see Vaughan, Joseph; 549-561

GLAHN, B.; Review of Forecast Verification: A Practitioner's Guide in Atmospheric Science, 607

Gosai, Ashmita; see Page, Cher M.; 1483-1489

GOTTSCHALCK, J.; see RoDELl, M.; 381-394

Graham, R.; see Palmer, T. N.; 853-872

Grant, Brad; see Mostek, Anthony; 823-829

GRANTHAM, D.; see CORMIER, R.; obituary, 1166

Graziani, Giovanni; see Rotach, Mathias W.; 1367-1385

Grazulis, T. P.; Review of The Forgotten Storm: The Great TriState Tornado of 1925,751

GregG, Michael; see Raymond, David J.; 1341-1354

Grell, GeorG; see DabBerdt, Walter F.; 563-586

GRIMMOND, SUE; see DABBERDT, WALTER F.; 563-586

Gross, James M.; see Landsea, Christopher W.; 1699-1712

GRUBIŠIC, VANDA; LEWIS, JoHN M.; Sierra Wave Project Revisited: 50 Years Later, 1127-1142

Gualdi, S.; see Palmer, T. N.; 853-872

GuÉrÉmy, J.-F.; see PALMER, T. N.; 853-872

Guest, P.; Review of Polar Lows: Mesoscale Weather Systems in the Polar Regions, 611 
Gumley, Liam E.; see Huang, Hung-Lung; 159-161

Gurtz, Joachim; see Rotach, Mathias W.; 1367-1385

Hagedorn, R.; see Palmer, T. N.; 853-872

Hanna, Steven; see Dabberdt, Walter F.; 563-586

Harris, Jasper L.; see GeER, Ira W.; 37-41

Hartmann, D. L.; “James Reed Holton, 1938-2004," obituary, 1015

Hayes, M. J.; see Pandya, R. E.; 425-430

Haylock, Malcolm R.; see Page, Cher M.; 1483-1489

Helsdon, John; see Lang, Timothy J.; 1107-1125

Hermann, M.; see Wendisch, M.; 89-91

Hermann, M.; see Wendisch, M.; ES1-ES8 (BAMS Online, January)

Hnilo, Justin J.; see Phillips, Thomas J.; 1903-1915

Holben, Brent N.; see Diner, David J.; 1491-1501

Holben, Brent N.; see KaHN, Ralph A.; 1511-1522

Holben, Brent N.; see Diner, David J.; 1535-1548

Hoshen, M.; see Palmer, T. N.; 853-872

Hostetler, Chris A.; see Diner, David J.; 1491-1501

Hostetler, Chris A.; see Diner, David J.; 1535-1548

Houser, P. R.; see Rodell, M.; 381-394

Howcroft, J. G.; Bedient, T.; Neilon, J.; Young-Wick, S.; Shuman, F.; "Harold Arthur Bedient, Jr., 1919-2003," obituary, 110

Hoyos, Carlos; see Webster, Peter J.; 1745-1765

Hristov, Tinomir; see Anderson, Kenneth; 1355-1365

Hu, Q.; Review of The Climate Near the Ground (Sixth Edition), 885

Hu, QI; FEnG, Song; U.S. Soil Temperature and Its Variation: A New Dataset, 29-31

Huang, Hung-Lung; Gumley, Liam E.; Strabala, Kathy; Li, Jun; Weisz, Elisabeth; Rink, Thomas; Baggett, Kevin C.; Davies, James E.; Smith, William L.; Dodge, James C.; International MODIS and AIRS Processing Package (IMAPP): A Direct Broadcast Software Package for the NASA Earth Observing System, 159-161

Hubbard, Kenneth G.; DeGaetano, Arthur T.; Robbins, Kevin D.; A Modern Applied Climate Information System, 811-812

Huebert, Barry J.; see Seinfeld, John H.; 367-380

InAPE, Kasis; see PAGe, Cher M.; 1483-1489

IRWIN, John; see DabBERDT, WALTER F.; 563-586

ISEMER, H.-J.; see LAWFord, R. G.; 1917-1930

JAMBOR, U.; see Rodell, M.; 381-394

JARVinen, Brian R.; see LANDSEA, Christopher W.; 1699-1712

Jensen, Michael L.; see Tiernström, Michael; 1305-1321

Jensen, Michael L.; see Tiernström, Michael; ES14-ES18 (BAMS Online, September)

Jewett, Brian; see Davis, Christopher; 1075-1093

Jin, MENGLIN; Analysis of Land Skin Temperature Using AVHRR Observations, 587-600

Johns, Robert; see Davis, Christopher; 1075-1093

Johnson, Mark E.; see Watson, Charles C., Jr.; 1713-1726

JOHnSON, R. M.; see PANDYA, R. E.; 425-430

Jolivet, D.; see Crewell, S.; 1565-1584

Jorgensen, David; see Davis, Christopher; 1075-1093

Kadygrov, Evgeny N.; see Rotach, Mathias W.; 1367-1385

Kadygrov, Vladislav; see Rotach, Mathias W.; 1367-1385

Kahn, Ralph A.; see Diner, David J.; 1491-1501

Kahn, Ralph A.; see Seinfeld, John H.; 1503-1509

Kahn, Ralph A.; Ogren, John A.; Ackerman, Thomas P.; Bösenberg, Jens; Charlson, Robert J.; Diner, David J.; Holben, Brent N.; Menzies, Robert T.; Miller, Mark A.; Seinfeld, John H.; Aerosol Data Sources and Their Roles within PARAGON, 1511-1522

Kahn, Ralph A.; see Ackerman, Thomas P.; 1523-1533

KaHn, Ralph A.; see Diner, David J.; 1535-1548

Kalkstein, Laurence S.; see Ebi, Kristie L.; 1067-1073

Kalkstein, Laurence S.; see Sheridan, Scott C.; 1931-1941

Kalnay, E.; see VukiceVic, T.; 48-49

KatzFey, JaCK; see Tselioudis, GeOrge; 830-835
Keenlyside, N.; see Palmer, T. N.; 853-872

Khelif, Diamal; see Anderson, Kenneth; 1355-1365

Kim, Jiyoung; see Seinfeld, JoHn H.; 367-380

KING, D. NT; Review of Global Change and Local Places:

Estimating, Understanding, and Reducing Greenhouse Gases, 1598

Knapp, Paul A.; Window of Opportunity: The Climatic Conditions of the Lewis and Clark Expedition of 1804-1806, 1289-1303

Knievel, Jason; see Davis, Christopher; 1075-1093

Knight, Charles; see Lang, Timothy J.; 1107-1125

Knupp, Kevin; see Davis, Christopher; 1075-1093

Koch, Steven E.; see Weckwerth, Tammy M.; 253-277

Koch, Steven E.; see Brennan, Michael J.; 935-939

Koch, Steven E.; see Benjamin, Stanley G.; 1871-1886

Koch, Steven E.; see Benjamin, Stanley G.; ES21-ES29 (BAMS Online, December)

Kocin, Paul J.; Uccellini, Louis W.; A Snowfall Impact Scale Derived from Northeast Storm Snowfall Distributions, 177-194

Kocin, Paul J.; Uccellini, Louis W.; A Snowfall Impact Scale Derived from Northeast Storm Snowfall Distributions, ES9ES10 (BAMS Online, February)

Koenig, Jane; see Vaughan, Joseph; 549-561

KoIKE, T.; see LAWFORD, R. G.; 1917-1930

Koracin, D.; see Lewis, J. M.; 395-408

Koracin, D.; see LEWIS, J.; obituary, 769

Kossin, James P.; Schubert, Wayne H.; Mesovortices in Hurricane Isabel, 151-153

Kousky, V. E.; Review of Global Change and Regional Impacts: Water Availability and Vulnerability of Ecosystems and Society in the Semiarid Northeast of Brazil, 1943

KRÄMER, M.; see WendisCH, M.; 89-91

KrÄMER, M.; see WendisCH, M.; ES1-ES8 (BAMS Online, January)

Krasnov, O. A.; see Crewell, S.; 1565-1584

Krehbiel, Paul; see Lang, Timothy J.; 1107-1125

Kubota, M.; see Curry, J. A.; 409-424

Kustas, William P.; see Diak, George R.; 65-78

Lackmann, Gary M.; see Brennan, Michael J.; 935-939

LaDue, Jim; see Mostek, Anthony; 823-829

LAMB, BRIAN; See VAUGHAN, JOSEPH; 549-561

LAMB, Brian; see DabBerdT, Walter F.; 563-586

Lamb, P. J.; Prospero, J. M.; “Eric Bradshaw Kraus, 1912-2003," obituary, 1783

LAMONI, R.; Review of Weather Rage, 1600

Landsea, Christopher; see Chenoweth, Michael; 1689-1697

Landsea, Christopher W.; Franklin, James L.; McAdie, Colin J.; Beven, John L., II; Gross, James M.; Jarvinen, Brian R.; Pasch, Richard J.; Rappaport, Edward N.; Dunion, Jason P.; Dodge, Peter P.; A Reanalysis of Hurricane Andrew's Intensity, $1699-1712$

Lang, Timothy J.; Miller, L. Jay; Weisman, Morris; Rutledge, Steven A.; Barker, Llyle J., III; Bringi, V. N.; Chandrasekar, V.; Detwiler, Andrew; Doesken, Nolan; Helsdon, John; Knight, Charles; Krehbiel, Paul; Lyons, Walter A.; MacGorman, Don; Rasmussen, Erik; Rison, William; Rust, W. David; Thomas, Ronald J.; The Severe Thunderstorm Electrification and Precipitation Study, 1107-1125

Latif, M.; see Palmer, T. N.; 853-872

Laurent, Victoire; see Page, Cher M.; 1483-1489

LaWford, R. G.; Stewart, R.; RoAds, J.; Isemer, H.-J.; Manton, M.; Marengo, J.; Yasunari, T.; Benedict, S.; Koike, T.; Williams, S.; Advancing Global- and Continental-Scale Hydrometeorology: Contributions of GEWEX Hydrometeorology Panel, 1917-1930

Lazar, A.; see Palmer, T. N.; 853-872

Leck, Caroline; see TJernström, Michael; 1305-1321

Leck, Caroline; see TJernström, Michael; ES14-ES18 (BAMS Online, September)

Lee, Wen-Chau; see Davis, Christopher; 1075-1093 
Legler, David M.; see Smith, Shawn R.; 979-994

LeMone, Margaret A.; see Weckwerth, Tammy M.; 253-277

Lemos, M. C.; Review of Late Victorian Holocausts: El Niño

Famines and the Making of the Third World, 93

Letro, S.; Review of Florida Weather (Second Edition), 1772

LeVIN, Z.; see WENDISCH, M.; 89-91

LeVIN, Z.; see WeNDISCH, M.; ES1-ES8 (BAMS Online, January)

Levinson, D. H., Editor; W Aple, A. M., Editor; State of the Climate in 2003, S1-S72 (June)

LeVizZani, Vincenzo; see Rosenfeld, Daniel; 873-881

Lewis, J. M.; Koracin, D.; Redmond, K. T.; Sea Fog Research in the United Kingdom and United States: A Historical Essay Including Outlook, 395-408

Lewis, J.; Koracin, D.; “Dale F. Leipper, 1914-2004,” obituary, 769

Lewis, J. M.; see Aune, R. M.; obituary, 770

LewIS, John M.; see GrubišIC, VANDA; 1127-1142

Li, Jun; see HuANG, Hung-Lung; 159-161

Lin, B.; see Curry, J. A.; 409-424

LiN, F. Y.; Moore, J. T.; “Gandikota V. Rao, 1934-2004,” obituary, 1781

LindSEY, DAn; see Mostek, Anthony; 823-829

Lindstrom, E. J.; Review of Oceans 2020: Science, Trends, and the Challenge of Sustainability, 284

LisT, RoLAND; Weather Modification-A Scenario for the Future, $51-63$

Liverman, D. M.; see Bales, R. C.; 1727-1734

LOHMANN, D.; see Rodell, M.; 381-394

LÖHNERT, U.; see CREWELL, S.; 1565-1584

LynCh, A. H.; Curry, J. A.; BrunNer, R. D.; MaslaniK, J. A.; Toward an Integrated Assessment of the Impacts of Extreme Wind Events on Barrow, Alaska, 209-221

Lyons, Walter A.; see LANG, Timothy J.; 1107-1125

MacGorman, Don; see Lang, Timothy J.; 1107-1125

Madronich, Sasha; see Dabberdt, Walter F.; 563-586

Maisonnave, E.; see Palmer, T. N.; 853-872

Maitrepierre, Luc; see Page, Cher M.; 1483-1489

Makmur, Erwin E. P.; see PAGe, Cher M.; 1483-1489

Manton, M.; see Lawford, R. G.; 1917-1930

Manton, Mike; see Page, Cher M.; 1483-1489

Marengo, J.; see LaWford, R. G.; 1917-1930

Markowicz, Krzysztof M.; see SeInfeld, John H.; 367-380

Marletto, V.; see Palmer, T. N.; 853-872

MartonchiK, John V.; see Diner, David J.; 1491-1501

Martonchik, John V.; see Ackerman, Thomas P.; 1523-1533

MASER, R.; see WENDISCH, M.; 89-91

MASER, R.; see WENDISCH, M.; ES1-ES8 (BAMS Online, January)

Maslanik, J. A.; see Lynch, A. H.; 209-221

Mass, ClifF; see Vaughan, JoSePH; 549-561

Mathieu, E.; see Wendisch, M.; 89-91

Mathieu, E.; see Wendisch, M.; ES1-ES8 (BAMS Online, January)

MAYKut, NAYdenE; see VAUGHAN, JosePh; 549-561

McAdie, Colin J.; see Landsea, Christopher W.; 1699-1712

McCarthy, James J.; see Epstein, Paul R.; 1863-1869

McDonnell, J. D.; see PANDYA, R. E.; 425-430

McFarquhar, Gregory; see Davis, Christopher; 1075-1093

McGree, Simon; see PAGe, Cher M.; 1483-1489

McHenry, John N.; Ryan, William F.; Seaman, Nelson L.; Coats, Carlie J., Jr.; Pudykiewicz, Janusz; Arunachalam, Sarav; VuKovich, JEFFery M.; A Real-Time Eulerian Photochemical Model Forecast System: Overview and Initial Ozone Forecast

Performance in the Northeast U.S. Corridor, 525-548

McMiluIN, L.; “David Q. Wark, 1918-2002," obituary, 108

MCNoldy, Brian D.; Triple Eyewall in Hurricane Juliette, 16631666

McPhaden, Michael J.; Evolution of the 2002/03 El Niño, 677-695

McQueen, JefF; see Dabberdt, Walter F.; 563-586

Meagher, James; see DabBerdt, Walter F.; 563-586

MeciKalski, John R.; see Diak, George R.; 65-78

Melani, Samantha; see Rosenfeld, Daniel; 873-881
MenG, C.-J.; see Rodell, M.; 381-394

Menzies, Robert T.; see Diner, David J.; 1491-1501

Menzies, Robert T.; see Kahn, Ralph A.; 1511-1522

Menzies, Robert T.; see Diner, David J.; 1535-1548

MeYER, S. J.; Review of Meteorology: Understanding the

Atmosphere, 1944

Meywerk, J.; see Crewell, S.; 1565-1584

Miller, Evgeny; see Rotach, Mathias W.; 1367-1385

Miller, L. Jay; see Lang, Timothy J.; 1107-1125

Miller, Mark A.; see Diner, David J.; 1491-1501

Miller, Mark A.; see Kahn, Ralph A.; 1511-1522

Miller, Mark A.; see Diner, David J.; 1535-1548

Mills, G.; Review of Cities and Climate Change: Urban

Sustainability and Global Environmental Governance, 883

Mills, Elizabeth W.; see Geer, Ira W.; 37-41

Mrtchell, K.; see Rodel, M.; 381-394

Moerman, Marcel; see Anderson, Kenneth; 1355-1365

Mogit, H. Michael; Cloud Stamps, 1247

Moore, J. T.; see LIN, F. Y.; obituary, 1781

Moore, James; see Davis, Christopher; 1075-1093

Moore, James A.; see WeCKWERTh, TAMmy M.; 253-277

Moran, Joseph M.; see Geer, Ira W.; 37-41

Morehouse, B. J.; see BaLes, R. C.; 1727-1734

Morse, A. P.; see Palmer, T. N.; 853-872

Mostek, Anthony; Weaver, John; Bikos, Dan; Lindsey, Dan; Zajac, Bard; BaChmeIER, SCOTT; WhitTAKer, Tom; MotTA, Brian;

Grant, Brad; LaDue, Jim; Ferree, John; VISIT: Bringing

Training to Weather Service Forecasters Using a New

Distance-Learning Tool, 823-829

Motta, Brian; see Mostek, Anthony; 823-829

Murillo, Shirley; see Powell, Mark; 845-851

Murnane, Richard J.; Climate Research and Reinsurance, 697-707

Murphey, Hanne V.; see Wakimoto, Roger M.; 1095-1106

Murphy, K. A.; see Pandya, R. E.; 425-430

Murray, A. B.; Review of Coasts: Form, Process, and Evolution, 1389

NACASS, P.; see WeNDISCH, M.; 89-91

NACASS, P.; see WENDISCH, M.; ES1-ES8 (BAMS Online, January)

Nakamigawa, Hiroshi; see Page, Cher M.; 1483-1489

Nath, Radhika; see Stewart, Thomas R.; 223-235

NeIlon, J.; see Howcroft, J. G.; obituary, 110

Neininger, BRUno; see Rotach, Mathias W.; 1367-1385

NeSE, L. M.; Review of The Pennsylvania Weather Book, 1008

Nicholls, Neville; see PAGe, Cher M.; 1483-1489

Nof, Doron; de Boer, Agatha M.; From the Southern Ocean to the North Atlantic in the Ekman Layer, 79-87

NoONE, K.; see Wendisch, M.; 89-91

NoOnE, K.; see WENDISCH, M.; ES1-ES8 (BAMS Online, January)

Norman, John M.; see Diak, George R.; 65-78

O’Brien, James J.; see Smith, SHAWn R.; 979-994

Odman, Talat; see DabberdT, W alter F.; 563-586

Ogren, John A.; see Diner, David J.; 1491-1501

Ogren, John A.; see Seinfeld, John H.; 1503-1509

Ogren, John A.; see KaHN, Ralph A.; 1511-1522

Ogren, John A.; see Diner, David J.; 1535-1548

Ohlmann, Carter; see Raymond, David J.; 1341-1354

OKe, T. R.; see Fischer, Kenneth W.; 725-732

Olson, Jerry G.; see Phillips, Thomas J.; 1903-1915

Oltmans, Samuel J.; see Thompson, AnNe M.; 1549-1564

Oncley, Steven P.; see Tiernström, Michael; 1305-1321

Oncley, SteVen P.; see TJernström, Michael; ES14-ES18 (BAMS Online, September)

Orfila, B.; see Palmer, T. N.; 853-872

Orlove, Bentamin S.; Broad, Kenneth; Petty, Aaron M.; Factors that Influence the Use of Climate Forecasts: Evidence from the 1997/98 El Niño Event in Peru, 1735-1743

Orlove, Benjamin S.; Broad, Kenneth; Petty, Aaron M.; Results of Logit Models, ES19-ES20 (BAMS Online, November)

OsBorne, S.; see WENDISCH, M.; 89-91 
Osborne, S.; see Wendisch, M.; ES1-ES8 (BAMS Online, January) OTterson, SAlLy; see Vaughan, JosePh; 549-561

Ouprasitwong, Nongnat; see Page, Cher M.; 1483-1489

Page, Cher M.; Nicholls, Neville; Plummer, Neil; Trewin, Blair; Manton, Mike; Alexander, Lisa; Chambers, Lynda E.; Choi, Youngeun; Collins, Dean A.; Gosai, Ashmita; Della-Marta, Paul; Haylock, Malcolm R.; Inape, Kasis; LaURent, Victoire; Maitrepierre, Luc; Makmur, Erwin E. P.; Nakamigawa, Hiroshi; Ouprasitwong, Nongnat; McGree, Simon; Pahalad, Janita; Salinger, M. J.; Tibig, Lourdes; Tran, Trong D.; Vediapan, Kaliapan; Zhai, Panmao; Data Rescue in the Southeast Asia and South Pacific Region: Challenges and Opportunities, 1483 1489

Pahalad, Janita; see Page, Cher M.; 1483-1489

Palmer, T. N.; Alessandri, A.; Andersen, U.; Cantelaube, P.;

Davey, M.; Délécluse, P.; Déqué, M.; Diez, E.; Doblas-Reyes, F.

J.; Feddersen, H.; Graham, R.; Gualdi, S.; Guérémy, J.-F.;

Hagedorn, R.; Hoshen, M.; Keenlyside, N.; Latif, M.; Lazar, A.;

Maisonnave, E.; Marletto, V.; Morse, A. P.; Orfila, B.; Rogel,

P.; Terres, J.-M.; Thomson, M. C.; Development of a European

Multimodel Ensemble System for Seasonal-to-Interannual

Prediction (DEMETER), 853-872

Pandya, R. E.; Smith, D. R.; Ramamurthy, M. K.; Croft, P. J.;

Hayes, M. J.; Murphy, K. A.; MCDonnell, J. D.; Johnson, R. M.;

FrIEDMAN, H. A.; 11th AMS Education Symposium, 425-430

Parsons, David B.; see WeCKWerth, Tammy M.; 253-277

Pasch, Richard J.; see Landsea, Christopher W.; 1699-1712

Paulson, Clayton; see Raymond, David J.; 1341-1354

Penner, Joyce E.; see Diner, David J.; 1491-1501

Penner, Joyce E.; see Ackerman, Thomas P.; 1523-1533

Perrie, W.; see Curry, J. A.; 409-424

Persson, P. Ola G.; see TJernström, Michael; 1305-1321

Persson, P. Ola G.; see TJernström, Michael; ES14-ES18 (BAMS Online, September)

Petersen, Walter A.; see Raymond, David J.; 1341-1354

Petty, Aaron M.; see Orlove, Benjamin S.; 1735-1743

Petty, Aaron M.; see Orlove, Benjamin S.; ES19-ES20 (BAMS Online, November)

Pfeilsticker, K.; see Crewell, S.; 1565-1584

Phillips, Thomas J.; Potter, Gerald L.; Williamson, David L.; Cederwall, Richard T.; Boyle, James S.; Fiorino, Michael; Hnilo, Justin J.; Olson, Jerry G.; XIE, Shaocheng; Yio, J. John; Evaluating Parameterizations in General Circulation Models: Climate Simulation Meets Weather Prediction, 1903-1915

PIELKE, R. A., SR.; Review of Global Environmental Change and Land Use, 1005

Pielke, Roger, JR.; see Stewart, Thomas R.; 223-235

Pleim, Jonathan; see Dabberdt, Walter F.; 563-586

Plummer, Neil; see Page, Cher M.; 1483-1489

Porter, William A.; see Geer, Ira W.; 37-41

Potter, Gerald L.; see Phillips, Thomas J.; 1903-1915

Powell, Mark; Bowman, David; Gilhousen, David; Murillo, Shirley; Carrasco, Nick; St. Fleur, Russell; Tropical Cyclone Winds at Landfall: The ASOS-C-MAN Wind Exposure Documentation Project, 845-851

Prospero, J. M.; see Lamb, P. J.; obituary, 1783

Przybylinski, Ron; see Davis, Christopher; 1075-1093

Pudykiewicz, Janusz; see McHenry, John N.; 525-548

QuANTE, M.; see Crewell, S.; 1565-1584

Quinn, Frank H.; see Assel, Raymond A.; 1143-1151

Quinn, Patricia K.; see Seinfeld, John H.; 367-380

RADAKOVICH, J.; see Rodell, M.; 381-394

Raga, Graciela B.; see Bretherton, Christopher S.; 967-977

Ramamurthy, M. K.; see Pandya, R. E.; 425-430

Rappaport, Edward N.; see Landsea, Christopher W.; 1699-1712

Rasch, Philip J.; see Diner, David J.; 1491-1501

Rasch, Philip J.; see Ackerman, Thomas P.; 1523-1533

Rasch, Philip J.; see Diner, David J.; 1535-1548

Rasmussen, Erik; see Lang, Timothy J.; 1107-1125
Rauber, Robert; see Davis, Christopher; 1075-1093

Raymond, David J.; Esbensen, Steven K.; Paulson, Clayton; GregG, Michael; Bretherton, Christopher S.; Petersen, Walter A.; Cifelli, Robert; Shay, Lynn K.; Ohlmann, Carter; Zuidema, PAQUITA; EPIC2001 and the Coupled Ocean-Atmosphere System of the Tropical East Pacific, 1341-1354

ReDmond, K. T.; see Lewis, J. M.; 395-408

REEDER, R. A.; see CURRY, J. A.; 409-424

Reid, JefFery S.; see Anderson, Kenneth; 1355-1365

Reising, Steven; see Anderson, KenNeth; 1355-1365

RENFreW, I. A.; see CuRRY, J. A.; 409-424

RIND, D.; Review of Methane Hydrates in Quaternary Climate

Change: The Clathrate Gun Hypothesis, 279

Rink, Thomas; see Huang, Hung-Lung; 159-161

Rison, William; see Lang, Timothy J.; 1107-1125

RoADS, J.; Experimental Weekly to Seasonal U.S. Forecasts with the Regional Spectral Model, 1887-1902

ROADS, J.; see LAWFORD, R. G.; 1917-1930

Robbins, Kevin D.; see Hubbard, Kenneth G.; 811-812

Robinson, LaWrence; see Ebi, Kristie L.; 1067-1073

Robinson, P.; Review of Issues in International Climate Policy, 431

Rodell, M.; Houser, P. R.; Jambor, U.; GotTschalck, J.; Mitchell, K.; Meng, C.-J.; Arsenault, K.; Cosgrove, B.; Radakovich, J.; Bosilovich, M.; Entin, J. K.; Walker, J. P.; Lohmann, D.; Toll, D.; The Global Land Data Assimilation System, 381-394

RoEBBer, P.; Review of Wisconsin's Weather and Climate, 433

Rogel, P.; see PALMER, T. N.; 853-872

Rosenfeld, Daniel; Cattani, Elsa; Melani, Samantha; Levizzani, VINCENZO; Considerations on Daylight Operation of 1.6- versus 3.7- $\mu \mathrm{m}$ Channel on NOAA and METOP Satellites, 873-881

Rossow, W. B.; DueÑas, E. N.; The International Satellite Cloud Climatology Project (ISCCP) Web Site: An Online Resource for Research, 167-172

Rossow, W. B.; see CurRY, J. A.; 409-424

Rossow, William B.; see Tselioudis, George; 830-835

Rotach, Mathias W.; Calanca, Pierluigi; Graziani, Giovanni; Gurtz, Joachim; Steyn, D. G.; Vogt, Roland; Andretta, Marco; Christen, Andreas; Cieslik, Stanislaw; Connolly, Richard; De Wekker, Stephan F. J.; Galmarini, Stefano; Kadygrov, Evgeny N.; Kadygrov, Vladislav; Miller, Evgeny; Neininger, Bruno; Rucker, Magdalena; Van Gorsel, Eva; Weber, Heidi; Weiss, Alexandra; Zappa, Massimiliano; Turbulence Structure and Exchange Processes in an Alpine Valley: The Riviera Project, 1367-1385

Rucker, Magdalena; see Rotach, Mathias W.; 1367-1385

Russell, Lynn M.; see Seinfeld, John H.; 367-380

Russell, Philip B.; see Seinfeld, John H.; 367-380

Rust, W. David; see Lang, Timothy J.; 1107-1125

Rutledge, Steven A.; see Lang, Timothy J.; 1107-1125

Ryan, William F.; see McHenry, John N.; 525-548

SAlinger, M. J.; see PAGE, Cher M.; 1483-1489

Schmeling, M.; Review of Atmospheric Chemistry in a Changing World: An Integration and Synthesis of a Decade of

Tropospheric Chemistry Research, 1006

Schmid, H. P.; Review of The Earth's Biosphere: Evolution, Dynamics, and Change, 745

Schmid, Hans Peter; see Dabberdt, Walter F.; 563-586

Schmidlin, Francis J.; see Thompson, AnNe M.; 1549-1564

SCHMIDT, S.; see CREWELL, S.; 1565-1584

SCHNEIDER, J.; see WENDISCH, M.; 89-91

SCHNEIDER, J.; see WeNDISCH, M.; ES1-ES8 (BAMS Online, January)

SCholl, T.; see Crewell, S.; 1565-1584

SCHRÖDER, M.; see CreWell, S.; 1565-1584

SCHUberT, Wayne H.; see Kossin, James P.; 151-153

SChUlTZ, DAvid M.; Historical Research in the Atmospheric Sciences: The Value of Literature Reviews, Libraries, and Librarians, 995-999

SCHUlZ, J.; see CURRY, J. A.; 409-424 
SCHÜTZ, L.; see WENDISCH, M.; 89-91

SchÜTZ, L.; see WendisCH, M.; ES1-ES8 (BAMS Online, January)

Schwartz, Barry E.; see Benjamin, Stanley G.; 1871-1886

Schwartz, Barry E.; see Benjamin, Stanley G.; ES21-ES29 (BAMS

Online, December)

Schwartz, STEPhen E.; see Diner, David J.; 1491-1501

Schwartz, Stephen E.; see SeInfeld, John H.; 1503-1509

SCHWARZENBÖCK, A.; see WENDISCH, M.; 89-91

Schwarzenböck, A.; see Wendisch, M.; ES1-ES8 (BAMS Online, January)

Scoggins, J. R.; “Vance Edwards Moyer, 1914-2003," obituary, 109

Seaman, Nelson L.; see McHenry, John N.; 525-548

Seinfeld, John H.; Carmichael, Gregory R.; Arimoto, Richard;

Conant, William C.; Brechtel, Frederick J.; Bates, Timothy S.; Cahill, Thomas A.; Clarke, Antony D.; Doherty, Sarah J.; Flatau, Piotr J.; Huebert, Barry J.; Kim, Jiyoung; Markowicz, Krzysztof M.; Quinn, Patricia K.; Russell, Lynn M.; Russell, Philip B.; Shimizu, Atsushi; Shinozuka, Yohei; Song, Chul H.; Tang, Youhua; Uno, Itsushi; Vogelmann, Andrew M.; Weber, Rodney J.; WoO, Jung-Hun; Zhang, XIAO Y.; ACE-ASIA: Regional Climatic and Atmospheric Chemical Effects of Asian Dust and Pollution, 367-380

Seinfeld, John H.; see Diner, David J.; 1491-1501

Seinfeld, John H.; Kahn, Ralph A.; Anderson, Theodore L.; Charlson, Robert J.; Davies, Roger; Diner, David J.; OGren, John A.; Schwartz, STephen E.; Wielicki, Bruce A.; Scientific Objectives, Measurement Needs, and Challenges Motivating the PARAGON Aerosol Initiative, 1503-1509

Seinfeld, John H.; see Kahn, Ralph A.; 1511-1522

Sellinger, Cynthia E.; see Assel, Raymond A.; 1143-1151

Servain, JaCQues; see Smith, Shawn R.; 979-994

Sharman, R.; "Morton G. Wurtele, 1919-2004," obituary, 894

Shay, Lynn K.; see Raymond, David J.; 1341-1354

Sheridan, Scott C.; KalKStein, Laurence S.; Progress in Heat

Watch-Warning System Technology, 1931-1941

Shimizu, Atsushi; see Seinfeld, John H.; 367-380

SHINDELl, D. T.; Review of The Atmospheric Environment: Effects of Human Activity, 613

Shinozuka, Yohei; see Seinfeld, John H.; 367-380

SHUMAN, F.; see HowCROFT, J. G.; obituary, 110

Simmer, C.; see Crewell, S.; 1565-1584

Sissenwine, M.; see Cormier, R.; obituary, 1166

SMith, D. R.; see PANDYA, R. E.; 425-430

Smith, Michael; see Anderson, Kenneth; 1355-1365

SMITH, S. R.; see Curry, J. A.; 409-424

Smith, Shawn R.; Servain, Jacques; Legler, David M.; Stricherz,

James N.; Bourassa, Mark A.; O’Brien, James J.; In Situ-Based

Pseudo-Wind Stress Products for the Tropical Oceans, 979-994

Smith, William L.; see Huang, Hung-Lung; 159-161

Smull, Bradley; see Davis, Christopher; 1075-1093

Solomon, Susan; Daniel, John S.; Lewis and Clark: Pioneering

Meteorological Observers in the American West, 1273-1288

Song, Chul H.; see Seinfeld, John H.; 367-380

Soto, Rodolfo Acuña; see Therrell, Matthew D.; 1263-1272

Stahle, David W.; see Therrell, Matthew D.; 1263-1272

STAMMER, D.; Review of Inverse Modeling of the Ocean and Atmosphere, 96

SteEnburgh, W. James; One Hundred Inches in One Hundred

Hours: The Complex Evolution of an Intermountain Winter Storm Cycle, 16-20

Stephens, Graeme L.; see Diner, David J.; 1491-1501

Stephens, Graeme L.; see Diner, David J.; 1535-1548

Stevermer, Amy J.; see Windham, Thomas L.; 42-47

STEWART, R.; see LAWFORD, R. G.; 1917-1930

Stewart, Thomas R.; Pielke, Roger, Jr.; Nath, RadhiKa;

Understanding User Decision Making and the Value of

Improved Precipitation Forecasts: Lessons from a Case Study, 223-235

Steyn, D. G.; see Rotach, Mathias W.; 1367-1385
St. Fleur, Russell; see Powell, Mark; 845-851

Strabala, Kathy; see Huang, Hung-Lung; 159-161

Strapp, J. Walter; see Abraham, James; 1323-1339

STRATMANN, F.; see Wendisch, M.; 89-91

Stratmann, F.; see Wendisch, M.; ES1-ES8 (BAMS Online, January)

Stricherz, James N.; see Smith, Shawn R.; 979-994

Swerdlin, Scott P.; see Warner, Thomas T.; 709-716

Szoke, Edward J.; see Benjamin, Stanley G.; 1871-1886

Szoke, Edward J.; see Benjamin, Stanley G.; ES21-ES29 (BAMS Online, December)

TAN, D. G. H.; see GÉrARD, E.; 237-251

TANG, Youhua; see SeINFELd, John H.; 367-380

TARgino, Admir; see TJernström, Michael; 1305-1321

TARGINO, Admir; see TJERnStröm, MichaEL; ES14-ES18 (BAMS

Online, September)

Teisberg, Thomas J.; see EbI, Kristie L.; 1067-1073

Terres, J.-M.; see Palmer, T. N.; 853-872

Terrill, Eric; see Anderson, Kenneth; 1355-1365

Therrell, Matthew D.; Stahle, David W.; Soto, Rodolfo Acuña; Aztec Drought and the "Curse of One Rabbit," 1263-1272

Thomas, Ronald J.; see Lang, Timothy J.; 1107-1125

Thompson, Anne M.; Witte, Jacquelyn C.; Oltmans, Samuel J.; Schmidlin, Francis J.; SHADOZ-A Tropical OzonesondeRadiosonde Network for the Atmospheric Community, 1549-1564

Thomson, M. C.; see Palmer, T. N.; 853-872

Tibig, Lourdes; see Page, Cher M.; 1483-1489

Tikuisis, PETER; Finger Cooling during Cold Air Exposure, 717723

Tjernström, Michael; Leck, Caroline; Persson, P. Ola G.; Jensen, Michael L.; Oncley, Steven P.; Targino, Admir; The Summertime Arctic Atmosphere: Meteorological Measurements during the Arctic Ocean Experiment 2001, 1305-1321

Tjernström, Michael; Leck, Caroline; Persson, P. Ola G.; Jensen, Michael L.; Oncley, Steven P.; TARgino, Admir; Experimental Equipment, ES14-ES18 (BAMS Online, September)

Toll, D.; see Rodell, M.; 381-394

TORN, RYAN D.; see DIAK, GEORGE R.; 65-78

Torres, OMAR; see Diner, David J.; 1491-1501

Torres, OMAr; see Diner, David J.; 1535-1548

Tran, Trong D.; see Page, Cher M.; 1483-1489

Trapp, Robert; see Davis, Christopher; 1075-1093

Trautmann, T.; see Crewell, S.; 1565-1584

Travis, Larry D.; see Diner, David J.; 1491-1501

Travis, Larry D.; see Diner, David J.; 1535-1548

Trewin, Blair; see Page, Cher M.; 1483-1489

Trier, Stanley; see Davis, Christopher; 1075-1093

Tselioudis, George; Rossow, William B.; Gentilcore, Anastasia N.; KatZFEY, JACK; The Data Integration for Model Evaluation Web Site: A One-Stop Shop for Model Evaluation, 830-835

Tsintikidis, Dimitris; see ANDERSON, KenNeth; 1355-1365

Uccellini, Louis W.; see Kocin, Paul J.; 177-194

UCCellini, Louis W.; see KocIN, PAUl J.; ES9-ES10 (BAMS Online, February)

Uno, Itsushi; see SeInfeld, John H.; 367-380

UtTal, TANeIL; see Bretherton, Christopher S.; 967-977

Van Gorsel, Eva; see Rotach, Mathias W.; 1367-1385

van Lammeren, A.; see Crewell, S.; 1565-1584

vaN MEIJGaARD, E.; see Crewell, S.; 1565-1584

Vaughan, JosePh; Lamb, Brian; Frei, Chris; Wilson, Rob; BoWMan, Clint; Figueroa-Kaminsky, Cristiana; Otterson, Sally; Boyer, Mike; Mass, Cliff; Albright, Mark; Koenig, Jane; Collingwood, Alice; Gilroy, Mike; Maykut; Naydene; A Numerical Daily Air Quality Forecast System for the Pacific Northwest, 549-561

Vediapan, Kaliapan; see Page, Cher M.; 1483-1489

Velden, Christopher S.; see Dunion, Jason P.; 353-365

Venema, V.; see Crewell, S.; 1565-1584 
Vogelmann, Andrew M.; see Seinfeld, John H.; 367-380

Vogt, Roland; see Rotach, Mathias W.; 1367-1385

Vonder HaAr, T.; see VukiceVIC, T.; 48-49

Vukicevic, T.; Kalnay, E.; Vonder HaAr, T.; The Need for a

National Data Assimilation Education Program, 48-49

Vukovich, Jefferr M.; see McHenry, John N.; 525-548

WADE, G. S.; see AUNE, R. M.; obituary, 770

W AKIMOTO, Roger; see DAvis, Christopher; 1075-1093

Wakimoto, Roger M.; Cai, Huaging; Murphey, Hanne V.; The

Superior, Nebraska, Supercell during BAMEX, 1095-1106

WALKER, J. P.; see RODELL, M.; 381-394

WANG, JUNHONG; see WeCKWERTH, TAMMY M.; 253-277

WAPLE, A. M., EDITOR; see Levinson, D. H., EdITOR; S1-S72 (June)

WARD, F.; Review of The Everything Weather Book: From Daily

Forecasts to Blizzards, Hurricanes, and Tornadoes-All You

Need to Know to Be Your Own Meteorologist, 1153

WARD, F.; Review of The Complete Idiot's Guide to Weather (Second Edition), 1153

WARD, F.; Review of The Coming Storm: The True Causes of Freak Weather-and Why It's Getting Worse, 1153

WARD, F.; Review of The Weather, 1153

Warner, Thomas T.; Bowers, James F.; Swerdlin, Scott P.; Beitler, Brian A.; A Rapidly Deployable Operational Mesoscale Modeling System for Emergency-Response Applications, 709-716

Watson, Charles C., Jr.; Johnson, Mark E.; Hurricane Loss Estimation Models: Opportunities for Improving the State of the Art, 1713-1726

Weaver, John; see Mostek, Anthony; 823-829

Weber, Heidi; see Rotach, Mathias W.; 1367-1385

Weber, Rodney J.; see SeINFELd, JoHn H.; 367-380

Webster, P. J.; see CURRY, J. A.; 409-424

Webster, Peter J.; Hoyos, Carlos; Prediction of Monsoon Rainfall and River Discharge on 15-30-Day Time Scales, 1745-1765

Weckwerth, Tammy M.; Parsons, David B.; Koch, Steven E.; Moore, James A.; LeMone, Margaret A.; Demoz, Belay B.; Flamant, Cyrille; Geerts, Bart; Wang, Junhong; Feltz, Wayne F.; An Overview of the International $\mathrm{H}_{2} \mathrm{O}$ Project (IHOP_2002) and Some Preliminary Highlights, 253-277

Weiter, Rodney F.; see Ebi, Kristie L.; 1067-1073

Weinbeck, Robert S.; see GeER, Ira W.; 37-41

Weisman, Morris; see Davis, Christopher; 1075-1093

Weisman, Morris; see LanG, Timothy J.; 1107-1125

Weiss, Alexandra; see Rotach, Mathias W.; 1367-1385

Weisz, Elisabeth; see Huang, Hung-Lung; 159-161

Weller, Robert A.; see Bretherton, Christopher S.; 967-977

WENDISCH, M.; COE, H.; BAUMGARDNER, D.; BRENGUIER, J.-L.;

Dreiling, V.; Fiebig, M.; Formenti, P.; Hermann, M.; Krämer,

M.; Levin, Z.; Maser, R.; Mathieu, E.; Nacass, P.; Noone, K.;

Osborne, S.; SChneider, J.; SchütZ, L.; SCHWarzenböck, A.;

Stratmann, F.; Wilson, J. C.; Aircraft Particle Inlets:

State-of-the-Art and Future Needs, 89-91
Wendisch, M.; Coe, H.; Baumgardner, D.; Brenguier, J.-L.; Dreiling, V.; Fiebig, M.; Formenti, P.; Hermann, M.; Krämer, M.; Levin, Z.; Maser, R.; Mathieu, E.; Nacass, P.; Noone, K.; Osborne, S.; SChneider, J.; SchÜtZ, L.; SChWARZenböCK, A.; Stratmann, F.; Wilson, J. C.; Aircraft Particle Inlets: State-of-the-Art and Future Needs, ES1-ES8 (BAMS Online, January)

Wendisch, M.; see CreWELl, S.; 1565-1584

Westphal, Douglas L.; see DabBerdt, Walter F.; 563-586

Westphal, James A.; see Garay, Michael J.; 1585-1594

WhITE, B.; "Morton Rubin, 1918-2004," obituary, 893

WhitTAKER, ToM; see Mostek, ANTHONY; 823-829

WICK, G. A.; see CURRY, J. A.; 409-424

Wielicki, Bruce A.; see Diner, DAvid J.; 1491-1501

Wielicki, Bruce A.; see SeInfeld, John H.; 1503-1509

Wielicki, Bruce A.; see Ackerman, Thomas P.; 1523-1533

Wielicki, Bruce A.; see Diner, David J.; 1535-1548

Willén, U.; see Crewell, S.; 1565-1584

Williams, S.; see LAWFord, R. G.; 1917-1930

Williamson, David L.; see Phillips, Thomas J.; 1903-1915

WILSON, J. C.; see WENDISCH, M.; 89-91

WiLson, J. C.; see WeNDISCH, M.; ES1-ES8 (BAMS Online, January)

WILSON, ROB; see VAUGHAN, JOSEPH; 549-561

Windham, Thomas L.; Stevermer, Amy J.; Anthes, Richard A.; SOARS: An Overview of the Program and Its First 8 Years, $42-47$

Winstanley, D.; Review of Global Climate: Current Research and Uncertainties in the Climate System, 98

Witiw, Michael R.; see Fischer, Kenneth W.; 725-732

Witte, Jacquelyn C.; see Thompson, AnNe M.; 1549-1564

Wolde, Mengistu; see Abraham, James; 1323-1339

Woo, Jung-Hun; see SeInfeld, John H.; 367-380

WOOD, RoberT; see Bretherton, CHristopher S.; 967-977

Wulfmeyer, V.; see Gérard, E.; 237-251

XIE, SHANG-PING; Satellite Observations of Cool OceanAtmosphere Interaction, 195-208

Xie, Shaocheng; see Phillips, Thomas J.; 1903-1915

Yasunari, T.; see LaWford, R. G.; 1917-1930

Yio, J. John; see Phillips, Thomas J.; 1903-1915

YounG-WICK, S.; see HowCroft, J. G.; obituary, 110

Yu, Bin; see Diner, David J.; 1491-1501

Yu, Bin; see ACKerman, Thomas P.; 1523-1533

Yuter, Sandra E.; see Bretherton, Christopher S.; 967-977

ZAJAC, BARD; see Mostek, ANTHONY; 823-829

Zappa, Massimiliano; see Rotach, Mathias W.; 1367-1385

ZENG, X.; Review of Large-Scale Atmosphere-Ocean Dynamics. Vol. I: Analytical Methods and Numerical Models; Vol. II: Geometric Methods and Models, 282

Zeng, X.; see CurRY, J. A.; 409-424

Zhai, PANmaO; see PAGe, Cher M.; 1483-1489

Zhang, Xiao Y.; see Seinfeld, John H.; 367-380

Ziegler, Conrad; see Davis, Christopher; 1075-1093

Zuidema, Paquita; see Raymond, David J.; 1341-1354
21 st Century Campaign, 1780, 1969

About our members, 26, 153, 350, 496, 768, 1012, 1162, 1396, $1602,1781,1974$

Annual meeting AMS honorary members, 895 at the annual meeting, 895 auditor's report, 1213 awards given at, 902 preliminary program, November

secretary-treasurer's report, 895

AMS Annual Report, March (supplement)

AMS Statement, 771

At Headquarters, 104, 621, 1399

Atmospheric policy program, 1777

Awards

AMS 75th Anniversary Scholarship, 1431, 1997

Abbe, 903 
AMS/Industry/Government Graduate Fellowships, 1403

AMS/Industry Minority Scholarships, 1421

AMS/Industry Undergraduate Scholarships, 1413

AMS Remote Sensing Prize, 1400

Anderson, 903

Applied Meteorology, 904

Bahethi Scholarship, 1434, 1998

Battan Author's, 904, 905

Baum Scholarship, 1432, 1998

Benton Scholarship, 1435, 1999

Biometeorology, 904

Broadcast Meteorology, 904

Brooks, 902

Charney, 902

Chapter, 906

Corporation, 904

Crow Scholarship, 1433, 1998

Editor's, 905, 906

Exceptional Specific Prediction, 903

Fellowships, 1403, 1983

Geotis Prize, 905

Glahn Scholarship, 1435, 1999

Grau Scholarship, 1430, 1997

Hagemeyer Scholarship, 1432, 1998

Hanks Scholarship, 1430, 1997

Hope Scholarship, 1433, 1998

Horton Lecturer, 905

Houghton, 903

Kreitzberg Scholarship, 1434, 1998

Meisinger, 903

Minority scholarships, 1421, 1992

Mitchell, 903

Murphy Scholarship, 1432, 1998

Orville Scholarship, 1430, 1997

Reichelderfer, 904

Rodriguez Scholarship, 1430, 1997

Rossby Research Medal, 902

Schroeder Scholarship, 1431, 1997

Special, 905

Stommel Research Award, 902

Student Chapter, 906

Suomi, 902

Sverdrup Gold Medal, 902

Teaching Excellence, 903

Undergraduate scholarships, 1413, 1986

Boards

Broadcast Meteorology, 1184

Bulletin of the American Meteorological Society, 1186

Certified Consulting Meteorologists, 1184

Continuing Education, 1184

Earth Interactions, 1186

flowchart of, 1180

Higer Education, 1190

Journal of Applied Meteorology, 1185

Journal of Atmospheric and Oceanic Technology, 1185

Journal of the Atmospheric Sciences, 1184

Journal of Climate, 1186

Journal of Hydrometeorology, 1186

Journal of Physical Oceanography, 1185

Meteorological and Geoastrophysical Abstracts, 1186

Meteorological Monographs, 1187

Monthly Weather Review, 1185

Operational Government Meteorologists, 1184

Outreach and Pre-College Education, 1190

Private Sector Meteorology, 1184

Urban Environment, 1190

Weather and Forecasting, 1185

Women and Minorities, 1190
Book excerpts

The Snowflake: Winter's Secret Beauty, 286

Sudden Sea: The Great Hurricane of 1938, 742

Climate Affairs: A Primer, 888

The Discovery of Global Warming, 1392

The Metamorphosis of a Geophysicist, 1946

Book issue, May

Calendar of meetings, 116, 304, 446, 624, 777, 907, 1020, 1194, $1438,1611,1789,2002$

Certified Consulting Meteorologists (CCM)

Board of, 1184

new, 767, 1396

Program, 1952

Chapter Channel

Anchorage, 813

Asheville, 1058

Central North Carolina, 157, 509, 814, 948

Chicago, 24, 509

Los Angeles, 24

North Carolina, 154

Omaha-Offutt, 1245

Packerland, 335, 1058

Southeast Arizona, 335

Twin Cities, 155, 509

Washington, D.C., 155

West Central Florida, 948, 1246

Chapters

affairs, 618, 1013

Commissions

Education and Human Resources, 1190

flowchart of, 1180

Planning, 1191

Professional Affairs, 1184

Publications, 1184

Scientific and Technological Activities (STAC), 1187

Committees

Admissions, 1179

Agricultural and Forest Meteorology, 1187

Annual Meeting Program, 1181

Applied Climatology, 1187

Artificial Intelligence Applications to Environmental Science, 1187

Atmospheric Chemistry, 1187

Atmospheric Electricity, 1187

Atmospheric and Oceanic Fluid Dynamics, 1187

Atmospheric Radiation, 1187

Aviation, Range, and Aerospace Meteorology, 1187

Atmospheric Research Awards, 1181

Awards Oversight, 1181

Biometeorology and Aerobiology, 1188

Boundary Layers and Turbulence, 1188

Climate Variability and Change, 1188

Cloud Physics, 1188

Coastal Environment, 1188

Development, 1181

Economic Development, 1182

Education Advisory, 1182

Ethics, 1182

Executive, 1181

Fellows, 1183

flowchart of, 1180

History of the Atmospheric Sciences, 1182

Hydrology, 1188

Information Systems, 1187

Intelligent Transportation Systems, 1182

Interaction of the Sea and Atmosphere, 1188

Interactive Information and Processing Systems, 1182

Investments, 1182

Judges for Undergraduate Awards, 1191 
Laser Atmospheric Studies, 1188

Local Chapter Affairs, 1182

Measurements, 1188

Mesoscale Processes, 1189

Meteorological Aspects of Air Pollution, 1189

Meteorology and Oceanography of the Southern Hemisphere, 1189

Middle Atmosphere, 1189

Mountain Meteorology, 1189

Nominating, 1182

Oceanographic Research Awards, 1183

of the Council, 1183

of the Executive Committee, 1179

Planned and Inadvertent Weather Modification, 1189

Polar Meteorology and Oceanography, 1189

Probability and Statistics, 1189

Public Policy, 1184

Radar Meteorology, 1189

Satellite Meteorology and Oceanography, 1190

Severe Local Storms, 1190

Societal Impacts, 1183

Tropical Meteorology and Tropical Cyclones, 1190

Weather Analysis and Forecasting, 1190

Conference notebook, 9, 143, 343, 498, 660, 943, 1059, 1252, 1477

Conferences, see Meetings and conference programs

Contents of AMS journals, see Journals, AMS, Tables of contents

Corporation and institutional members, 124, 307, 464, 636, 788, 918, 1030, 1202, 1446, 1620, 1828, 2008

Council

committees of, 1183

members, 1179

minutes of, 291

Directory

AMS, 1179

professional, 126, 309, 466, 638, 790, 920, 1032, 1204, 1448, $1622,1830,2010$

Education

Han Receives the 2004-05 AMS Graduate Fellowship in the History of Science, 1777

Essay, 736, 740, 1247

Executive Committee, 1181

Fellows of AMS

Committee on, 1183

elected for 2004, 616

Fellowships, Graduate, 1273

From the archives, 735

Forum

Historical Research in the Atmospheric Sciences: The Value of

Literature Reviews, Libraries, and Librarians, 995

Assessing Climate Stability, 1863

Groundwork,

U.S. Soil Temperature and Its Variation: A New Dataset, 29

History Books, 733

Honorary Members of AMS

new, 895

Image of the month, 820

In Box,

Enhancing Diversity in the Geosciences through National Dissemination of the AMS Online Weather Studies Distance Learning Course, 37

SOARS: an Overview of the Program and Its First 8 Years, 42

The Need for a National Data Assimilation Education

Program, 48

The International Satellite Cloud Climatology Project (ISCCP)

Web Site: An Online Resource for Research, 167

Making the Jump to the Private Sector, 173

VISIT: Bringing Training to Weather Service Forecasters Using a New Distance-Learning Tool, 823
The Data Integration for Model Evaluation Web Site: A OneStop Shop for Model Evaluation, 830

Data Rescue in the Southeast Asia and South Pacific Region: Challenges and Opportunities, 1483

In memoriam, 1606

In the field,

A Micrometeorological Investigation of a Restored California Wetland Ecosystem, 1170

International Science and Engineering Affiliated Fairs, summary of AMS participation in,

Investments Committee, 1182

Journals, AMS, see also Publications

Bulletin of the AMS, editors of, 1186

Earth Interactions, Board of, 1186

Journal of Applied Meteorology, Board of, 1185

Journal of Atmospheric and Oceanic Technology, Board of, 1185

Journal of the Atmospheric Sciences, Board of, 1184

Journal of Climate, Board of, 1186

Journal of Hydrometeorology, Board of, 1186

Journal of Physical Oceanography, Board of, 1185

Meteorological and Geoastrophysical Abstracts, Board of, 1186

Meteorological Monographs, Board of, 1187

Monthly Weather Review, Board of, 1185

Weather and Forecasting, Board of, 1185

Letter from headquarters, $101,289,439,615,765,889,1011$, $1161,1395,1601,1775,1951$

Local science fair results, 1977

Map Room, The, 16, 337, 935, 1657

Meet the AMS

Nathans, Jinny, 102

Kassas, Nahia, 290

Snow, John T., 440

Friedman, Mike, 620

Toohey-Morales, John, 766

Schein, Andrea, 891

Needham, Gretchen, 1397

Eosco, Gina, 1603

McVehil, George, 1778

Huntington, Marjorie, 1973

Meeting Summaries (of AMS sponsored or cosponsored meetings)

$11^{\text {th }}$ AMS Education Symposium, 425

$11^{\text {th }}$ Conference on Mountain Meteorology, 632

$13^{\text {th }}$ Conference on the Applications of Air Pollution

Meteorology with the Air and Waste Management Association, 1026

$13^{\text {th }}$ Conference on Interactions of the Sea and Atmosphere, 783

$16^{\text {th }}$ Conference on Biometeorology and Aerobiology, 1026

$16^{\text {th }}$ Symposium on Boundary Layers and Turbulence, 783

$26^{\text {th }}$ Conference on Agricultural and Forest Meteorology, 1026

Fifth Conference on the Urban Environment, 1026

Meetings of AMS, see Calendar of

Members

commissions, boards, and committees, 1179

corporation and institution, see Corporation and institutional members

deceased, see Obituaries

elected for 2004,616

new, 895

new, lists of, 112, 774, 1018, 1608

see, About our

Minutes, 291

New Publications, 94, 280, 432, 608, 746, 884, 1006, 1154, 1388, $1596,1768,1944$

News and Notes, 20, 148, 327, 489, 655, 807, 939, 1047, 1241, $1465,1643,1847$

News from our chapters, see Chapter news 
Notices of AMS, see American Meteorological Society, notices of Obituaries,

Bandeen, William Reed, 1604

Bedient, Harold Arthur, Jr., 110

Conover, John Hoagland, 441

Holton, James Reed, 1015

Kraus, Eric Bradshaw, 1783

Leipper, Dale F., 769

Marroquin, Adrian, 1016

Moyer, Vance Edwards, 109

Rao, Gandikota V., 1781

Raymond, William H., 770

Roberts, Michael Anderson, Jr., 1607

Rubin, Morton, 893

Sissenwine, Norm, 1166

Wark, David Q., 108

Wurtele, Morton G., 894

Observations, 151

Officers and councilors

list, 1179

Papers of note, 31, 161, 332, 492, 669, 817, 949, 1053, 1471, 1648

Photo Essay, 737

Picture of the month, 1655

Planning Commission, 1191

Potential Energy

Russell Schneider: The Making of a Meteorologist, 512

Grad School Advice: A Q\&A with Johannes Verlinde, 513

Dense Fog Climatology: Chicago O'Hare International Airport July 1996-April 2002, 515

Summer Internships: Helping Students Chart a Career Path, 517

Internships: At Television and Radio Stations, 518

RISE: Undergraduates Find that Regime Changes in Lorenz's

Model Are Predictable, 520

Professional Affairs, Commission on, 1184

Professional directory, see Directory, professional

Publications,

NOAA Central Library and AMS to Provide Access to Historic Monthly Weather Review Issues online, 259

The Journal of the American Meteorological Society, 1093

Publishers' addresses, 758

Reanalysis, 97, 284, 436, 752, 886, 1009, 1158, 1599, 1770

Reviews of books (listed by author of book)

Ackerman, S. A., and J. A. Knox, Meteorology: Understanding the Atmosphere, 1944

Akin, W., The Forgotten Storm: The Great Tri-State Tornado of 1925, 751

Aron, R. H., see Walker, G.

The Association of American Geographers Global Change and Local Places Research Team, Global Change and Local

Places: Estimating, Understanding, and Reducing

Greenhouse Gases, 1598

Behl, R. J., see Kennett, J. P.

Behre, K.-E., Ed., see Wefer, G., Ed.

Bennett, A. F., Inverse Modeling of the Ocean and Atmosphere, 96

Berger, W. H, Ed., see Wefer, G., Ed.

Betsill, M. M., see Bulkeley, H.

Brasseur, G. P., R. G. Prinn, and A. A. P. Pszenny, Atmospheric Chemistry in a Changing World: An Integration and Synthesis of a Decade of Tropospheric Chemistry Research, 1006

Bulkeley, H., and M. M. Betsill, Cities and Climate Change: Urban Sustainability and Global Environmental Governance, 883

Cannariato, K. G., see Kennett, J. P.

Cantrell, M., The Everything Weather Book: From Daily Forecasts to Blizzards, Hurricanes, and Tornadoes-All You Need to Know to Be Your Own Meteorologist, 1153
Collier, M., and Webb, R. H., Floods, Droughts, and Climate Change, 437

Comin, F. A., Ed., see Rodó, X. , Ed.

Davis, M., Late Victorian Holocausts: El NiZo Famines and the Making of the Third World, 93

de Araujo, J. C., Ed. see Gaiser, T., Ed.

Dolman, A. J., A. Verhagen, and C. A. Rovers, Eds., Global Environmental Change and Land Use, 1005

Etter, P. C., Underwater Acoustic Modeling and Simulation (Third Edition), 435

Field, J. G., G. Hempel, and C. P. Summerhayes, Eds., Oceans 2020: Science, Trends, and the Challenge of Sustainability, 284

Frischkorn, H., Ed., see Gaiser, T., Ed.

Gaiser, T., M. Krol, H. Frischkorn, and J. C. de Araujo, Eds., Global Change and Regional Impacts: Water Availability and Vulnerability of Ecosystems and Society in the Semiarid Northeast of Brazil, 1943

Gelber, B., The Pennsylvania Weather Book, 1008

Goldstein, M., The Complete Idiot's Guide to Weather (Second Edition), 1153

Griffin, see Winsberg, M. D.

Gupta, J., see Van Ierland, E. C.

Hempel, G., Ed., see Field, J. G., Ed.

Hendy, I. L., see Kennett, J. P.

Hewitt, C. N., and A. Jackson, Handbook of Atmospheric Science: Principles and Applications, 1391

Hopkins, E. J., Ed., see Moran, J. M., Ed.

Jackson, A., see Hewitt, C. N.

Jolliffe, I. T., and D. B. Stephenson, Forecast Verification: A Practitioner's Guide in Atmospheric Science, 607

Keim, B. D., see Zielinski, G. A.

Kennett, J. P., K. G. Cannariato, I. L. Hendy, and R. J. Behl, Methane Hydrates in Quaternary Climate Change: The Clathrate Gun Hypothesis, 279

Knox, J. A., see Ackerman, S. A.

Kok, M. T. J., see Van Ierland, E. C.

Krol, M., Ed., see Gaiser, T., Ed.

Libbrecht, K., and P. Rasmussen, The Snowflake: Winter's Secret Beauty, 1387

Libbrecht, K., and P. Rasmussen, The Snowflake: Winter's Secret Beauty, 1387

Lynch, J., The Weather, 1153

Marzano, F. S., and G. Visconti, Remote Sensing of Atmosphere and Ocean from Space: Models, Instruments and Techniques, 1595

Maslin, M., The Coming Storm: The True Causes of Freak Weather-and Why It's Getting Worse, 1153

McElroy, M. B., The Atmospheric Environment: Effects of Human Activity, 613

Moran, J. M., and Hopkins, E. J., Wisonsin's Weather and Climate, 433

Nese, J., and G. Schwartz, The Philadelphia Area Weather Book: Including Delaware, the Poconos, and the New Jersey Shore, 1769

Norbury, J., and I. Roulstone, Eds., Large-Scale AtmosphereOcean Dynamics. Vol. I: Analytical Methods and Numerical Models; Vol. II: Geometric Methods and Models, 282

O’Brien, J., see Winsberg, M. D.

Prinn, R. G., see Brasseur, G. P.

Pszenny, A. A. P. see Brasseur, G. P.

Rasmussen, E. A., Polar Lows: Mesoscale Weather Systems in the Polar Regions, 611

Rasmussen, P., see Libbrecht, $\mathrm{K}$.

Reynolds, R., Weather Rage, 1600

Rodó, X., and Comin, F. A., Eds., Global Climate: Current

Research and Uncertainties in the Climate System, 98

Roulstone, I., see Norbury, J.

Rovers, C. A., see Dolman, A. J., Ed. 
Schwartz, G., see Nese, J.

Smil, V., The Earth's Biosphere: Evolution, Dynamics, and Change, 745

Soon, W. W.-H., and S. H. Yaskell, The Maunder Minimum and the Variable Sun-Earth Connection, 1767

Stephenson, D. B., see Jolliffe, I. T.

Summerhayes, C. P., Ed., see Field, J. G., Ed.

Todhunter, P., see Walker, G.

Van Ierland, E. C., J. Gupta, and M. T. J. Kok, Issues in International Climate Policy, 431

Verhagen, A., Ed., see Dolman, A. J., Ed.

Visconti, G., see Marzano, F. S.

Walker, G., R. H. Aron, and P. Todhunter, The Climate Near the Ground (Sixth Edition), 885

Webb, R. H., see Collier, M.

Wefer, G., W. H. Berger, and K.-E. Behre, Eds., Climate Development and History of the North Atlantic Realm, 750

Winsberg, M. D., J. O'Brien, D. Zierden, and M. Griffin, Florida Weather (Second Edition), 1772

Woodroffe, C. D., Coasts: Form, Process and Evolution, 1389

Yaskell, S. H., see Soon, W. W.-H.

Zielinski, G. A., and B. D. Keim, New England Weather, New England Climate, 754

Zierden, see Winsberg, M. D.

Reviews of books (listed by title of book)

Atmospheric Chemistry in a Changing World: An Integration and Synthesis of a Decade of Tropospheric Chemistry Research, G. P. Brasseur, R. G. Prinn, and A. A. P. Pszenny, 1006

The Atmospheric Environment: Effects of Human Activity, M. B. McElroy, 613

Cities and Climate Change: Urban Sustainability and Global Environmental Governance, H. Bulkeley, and M. M. Betsill, 883

Climate Development and History of the North Atlantic Realm, G. Wefer, W. H. Berger, and K.-E. Behre, 750

The Climate Near the Ground (Sixth Edition), G. Walker, R. H. Aron, and P. Todhunter, 885

Coasts: Form, Process and Evolution, C. D. Woodroffe, 1389

The Coming Storm: The True Causes of Freak Weather-and Why It's Getting Worse, M. Maslin, 1153

The Complete Idiot's Guide to Weather (Second Edition), M. Goldstein, 1153

The Earth's Biosphere: Evolution, Dynamics, and Change, V. Smil, 745

The Everything Weather Book: From Daily Forecasts to Blizzards, Hurricanes, and Tornadoes-All You Need to Know to Be Your Own Meteorologist, M. Cantrell, 1153

Floods, Droughts, and Climate Change, M. Collier, and R. H. Webb, 437

Florida Weather (Second Edition), M. D. Winsberg, J. O'Brien D. Zierden, and M. Griffin, 1772

Forecast Verification: A Practitioner's Guide in Atmospheric Science, I. T. Jolliffe, and D. B. Stephenson, 607

The Forgotten Storm: The Great Tri-State Tornado of 1925, W. Akin, 751

Global Change and Local Places: Estimating, Understanding, and Reducing Greenhouse Gases, The Association of American Geographers Global Change and Local Places Research Team, 1598

Global Environmental Change and Land Use, A. J. Dolman, A. Verhagen, and C. A. Rovers, Eds., 1005

Global Change and Regional Impacts: Water Availability and Vulnerability of Ecosystems and Society in the Semiarid Northeast of Brazil, T. Gaiser, M. Krol, H. Frischkorn, and J. C. de Araujo, Eds., 1943

Global Climate: Current Research and Uncertainties in the Climate System, X. Rodó, and F. A. Comin, Eds., 98
Handbook of Atmospheric Science: Principles and Applications, C. N. Hewitt, and A. Jackson, 1391

Inverse Modeling of the Ocean and Atmosphere, A. F. Bennett, 96

Issues in International Climate Policy, E. C. Van Ierland, J. Gupta, and M. T. J. Kok, 431

Large-Scale Atmosphere-Ocean Dynamics. Vol. I: Analytical Methods and Numerical Models; Vol. II: Geometric Methods and Models, J. Norbury, and I. Roulstone, Eds., 282

Late Victorian Holocausts: El NiZo Famines and the Making of the Third World, M. Davis, 93

The Maunder Minimum and the Variable Sun-Earth Connection, W. W.-H. Soon, and S. H. Yaskell, 1767

Meteorology: Understanding the Atmosphere, S. A., Ackerman, and J. A. Knox, 1944

Methane Hydrates in Quaternary Climate Change: The Clathrate Gun Hypothesis, J. P. Kennett, K. G. Cannariato, I. L. Hendy, and R. J. Behl, 279

New England Weather, New England Climate, G. A. Zielinski, and B. D. Keim, 754

Oceans 2020: Science, Trends, and the Challenge of Sustainability, J. G. Field, G. Hempel, and C. P. Summerhayes, Eds. 284

The Pennsylvania Weather Book, B. Gelber 1008

The Philadelphia Area Weather Book: Including Delaware, the Poconos, and the New Jersey Shore, J. Nese, and G. Schwartz, 1769

Polar Lows: Mesoscale Weather Systems in the Polar Regions, E. A. Rasmussen, 611

Remote Sensing of Atmosphere and Ocean from Space: Models, Instruments and Techniques, F. S. Marzano, and G. Visconti, 1595

The Snowflake: Winter's Secret Beauty, K. Libbrecht, and P. Rasmussen, 1387

Underwater Acoustic Modeling and Simulation (Third Edition), P. C. Etter, 435

The Weather, J. Lynch, 1153

Weather Rage, R. Reynolds, 1600

Wisonsin's Weather and Climate, J. M. Moran, and E. J. Hopkins, 433

Scholarships

AMS Undergraduate, 1429

AMS/Industry Graduate, 1403

AMS/Industry Undergraduate, 1416

Minority, 1421

Science Fairs, 1977

Scientific and Technological Activities Commission, 1187

Seal of approval

radio seals of approval, list of, 1163

television seals of approval, list of, 103, 294, 441, 617, 771, $1014,1167,1397$

State of the Climate in 2003, S1 (June)

Statements

AMS, 297

of ownership, management, and circulation, November

Supplement

Climate Assessment for 2001, June

Survey, "Student Chapters of the AMS,"

Symposium, The Richard Reed Symposium,

Systems

International MODIS and AIRS Processing Package (IMAPP) A Direct Broadcast Software Package for the NASA Earth Observing System, 159

Undergraduate Awards and scholarships, see Awards

Committee of Judges for, 1191

Women and Minorities Board on, 1190 